Lei $\mathrm{Xi}$ and Chunqing Yang*

\title{
Evaluation of alpha-L-fucosidase for the diagnosis of hepatocellular carcinoma based on meta- analysis
}

https://doi.org/10.1515/labmed-2019-0152

Received September 3, 2019; accepted June 15, 2020; published online August 7, 2020

\begin{abstract}
Objectives: The main aim of the present study was to assess the diagnostic value of alpha-L-fucosidase (AFU) for hepatocellular carcinoma (HCC).

Methods: Studies that explored the diagnostic value of AFU in HCC were searched in EMBASE, SCI, and PUBMED. The sensitivity, specificity, and DOR about the accuracy of serum AFU in the diagnosis of HCC were pooled. The methodological quality of each article was evaluated with QUADAS-2 (quality assessment for studies of diagnostic accuracy 2). Receiver operating characteristic curves (ROC) analysis was performed. Statistical analysis was conducted by using Review Manager 5 and Open Meta-analyst.

Results: Eighteen studies were selected in this study. The pooled estimates for AFU vs. $\alpha$-fetoprotein (AFP) in the diagnosis of HCC in 18 studies were as follows: sensitivity of $0.7352(0.6827,0.7818)$ vs. $0.7501(0.6725,0.8144)$, and specificity of $0.7681(0.6946,0.8283)$ vs. $0.8208(0.7586$, 0.8697 ), diagnostic odds ratio (DOR) of $7.974(5.302,11.993$ ) vs. $13.401(8.359,21.483)$, area under the curve (AUC) of 0.7968 vs. 0.8451 , respectively.
\end{abstract}

Conclusions: AFU is comparable to AFP for the diagnosis of HCC.

Keywords: alpha-L-fucosidase (AFU); diagnosis; hepatocellular carcinoma (HCC); meta-analysis; $\alpha$-fetoprotein (AFP).

Lei $\mathrm{Xi}$ and Chunqing Yang contributed equally to this work.

*Corresponding author: Chunqing Yang, Department of Pathology, The First Affiliated Hospital of Nanjing Medical University, Nanjing, P.R. China, 210029, E-mail: 1399391865@qq.com Lei Xi, Department of Pathology, The First Affiliated Hospital of Nanjing Medical University, Nanjing, P.R. China
Introduction

In 2018, liver cancer morbidity and mortality ranked seventh and third among global tumors, respectively [1]. The number of new cases of liver cancer was 841,080 and the number of related deaths was 781,631. Hepatocellular carcinoma (HCC) is associated with chronic hepatitis B virus and chronic hepatitis $\mathrm{C}$ virus infection [2]. Due to the asymptomatic nature of early HCC and the lack of effective diagnostic and screening strategies, most patients $(>80 \%)$ present with advanced HCC stages [3]. A lot of cases have shown that early detection of HCC and timely treatment is critical for improving patient survival. Tumor markers are pivotal tools for the early diagnosis of tumors. $\alpha$-Fetoprotein (AFP) is a major marker widely used in the clinic for the detection of HCC [4]. However, AFP shows poor sensitivity and specificity in the early stages of HCC. Ba et al. (2012) revealed that AFP levels are not elevated in some HCC patients, while they were elevated in other patients with benign liver disease [5]. New biomarkers are urgently needed for specific early diagnosis of HCC patients.

Many groups have proposed alpha-L-fucosidase (AFU) as a tumor marker for the diagnosis of HCC. AFU is a lysosomal enzyme found in all mammalian cells and its activity in HCC patients was reported as significantly higher than that in patients with benign liver disease [6]. However, due to inconsistency among reports, diagnostic value of AFU is still not widely accepted. In this paper, we report a meta-analysis based on the results of published studies. The main aim is to assess the diagnostic value of AFU for HCC compared to AFP.

\section{Methods}

\section{Inclusion criteria of the study}

(1) No HCC treatment before sample collection, (2) the diagnostic values of AFU and AFP were tested in the same HCC patients, (3) sensitivity and specificity of AFP and AFU are reported or can be obtained by calculation, (4) patients of the control group had only other malignant liver cancer 
or benign liver disease, (5) all patients in the case group were diagnosed as HCC by gold standard assessment.

\section{Exclusion criteria of the study}

(1) The sample is from a tissue or another body fluid (i.e., not a serum sample), (2) the patient received HCC treatment before sampling (3) data on specificity and sensitivity are not available in the article, (4) animal study, (5) only had healthy people in control groups, or had no control group in studies.

\section{Search and selection}

The keywords selected included "alpha-L-fucosidase", "AFU", "hepatocellular carcinoma”, "HCC”, " $\alpha$-fetoprotein", and "AFP”. Keywords search and free words search were adopted. Papers published in English were searched in EMBASE, SCI, and PUBMED, with no limit in year of publication. In addition, the references in the included papers were searched and analyzed.

The two authors (Lei Xi and Chunqing Yang) independently extracted data from the selected articles and solved any discrepancy through discussion. The following data came from each article: first author, publication year, country, AFU test method, number of HCC and control, gender ratio and average age of HCC patients, pathological stage and raw data (true positive(TP), false positive(FP), false negative(FN), and true negative(TN) subjects).

\section{Evaluation of study quality}

The quality of each article was evaluated with the new assessment tool, Quality Assessment Diagnostic Accuracy Study 2 (QUADAS-2). QUADAS-2 has two components: risk of bias and applicability concerns. The risk of bias includes four parts: patient selection, index testing, reference standard, flow and timing [7]. The applicability concerns include the first three parts of the risk of bias.

\section{Data analysis}

Open Meta-Analyst and Review Manager 5 software were used for statistical analysis [8, 9]. First, a heterogeneity test was conducted. Heterogeneity produced by threshold effects was analyzed by Spearman coefficient and received operational characteristic (ROC) plots [10]. If the
Spearman's value was about 1 and $p<0.05$, a threshold effect was concluded. The heterogeneity index $\mathrm{I}^{2}$ produced by the non-threshold effect was examined through the inconsistency test and statistical significance was concluded in case of $\mathrm{I}^{2}>0$.

Meta-regression was used to analyze whether heterogeneity is caused by non-threshold effects. $p$-value $<0.05$ was considered statistically significant. The plotted ROC curve was used to calculate the AUC and Q value. Sensitivity, specificity, and DOR were obtained using the diagnostic random effects model and the DerSimonian-Laird method.

\section{Results}

\section{Search results}

The search retrieved 169 related articles. After removing the doublets, 86 articles remained, of which 23 were eligible for full-text evaluation. The detailed flow chart of the article selection is shown in Figure 1. Five articles were excluded based on not fulfilling inclusion and exclusion criteria and the remaining 18 articles [11-28] were selected in this study. The features of the selected articles are shown in Tables 1 and 2. These articles involved a total of 2,224 patients, of which 1,053 patients with HCC were in the experimental group and 1,171 patients without HCC were in the control group.

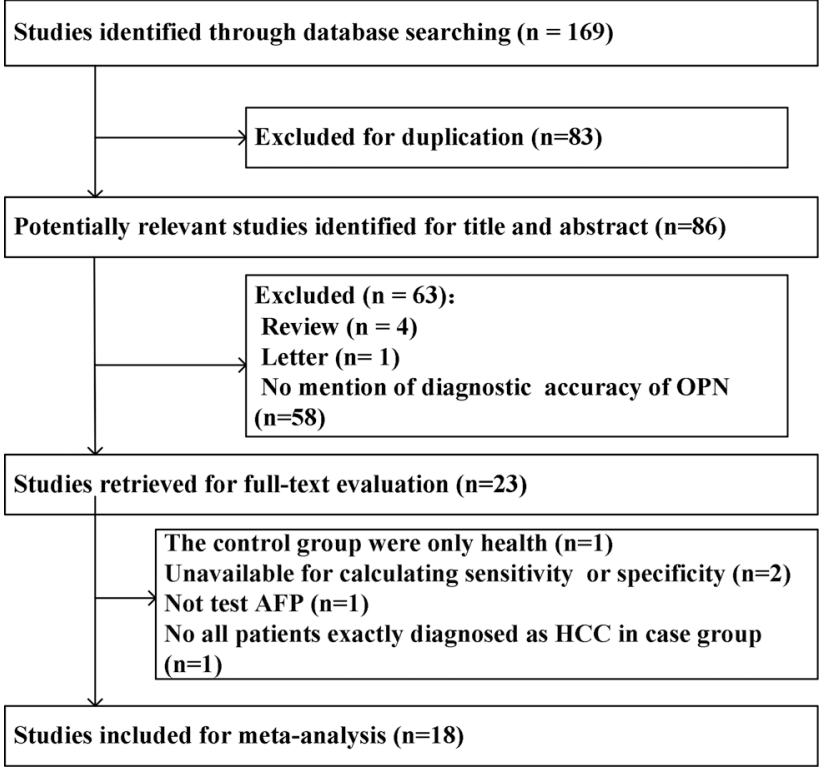

Figure 1: Article selection process. 
Table 1: Main features of the articles selected.

\begin{tabular}{|c|c|c|c|c|c|c|c|}
\hline Author & Year & Country & Test method & $\mathrm{HCC} /$ control & Gender (M/F) & Average age, years & Stage \\
\hline El-houseini & 2001 & Egypt & PNPF & $50 / 50$ & $37 / 11$ & 63.7 & Child-Pugh \\
\hline El-houseini & 2005 & Egypt & PNPF & $44 / 20$ & $34 / 10$ & 55 & TNM \\
\hline Shao & 2009 & China & PNPF & $30 / 30$ & $26 / 4$ & 51.2 & $\mathrm{~N}$ \\
\hline Zhu, J & 2013 & China & CNPF & $113 / 102$ & $95 / 18$ & 55.3 & $\mathrm{~N}$ \\
\hline Zhang & 2015 & China & PNPF & $116 / 104$ & $75 / 41$ & 57.8 & $\mathrm{~N}$ \\
\hline Wang & 2014 & China & CNPF & $459 / 210$ & $406 / 53$ & 51 & $\mathrm{BCLC}$ \\
\hline Zhu, JN & 2017 & China & CNPF & $36 / 36$ & $21 / 15$ & 50.2 & $\mathrm{~N}$ \\
\hline Xing & 2019 & China & PNPF & $325 / 187$ & $157 / 30$ & 53.7 & Child-Pugh \\
\hline Montaser & 2012 & Egypt & PNPF & $40 / 40$ & $33 / 7$ & 54.88 & Child-Pugh \\
\hline El-tayeh & 2012 & Egypt & PNPF & $37 / 59$ & $29 / 8$ & 55 & TNM \\
\hline Habachi & 2018 & Egypt & PNPF & $86 / 89$ & $\mathrm{~N}$ & $\mathrm{~N}$ & Child-Pugh \\
\hline Mossad & 2014 & Egypt & PNPF & $40 / 30$ & $14 / 26$ & 57.3 & Child-Pugh \\
\hline Bukofzer & 1989 & South Africa & PNPF & $72 / 64$ & $\mathrm{~N}$ & $\mathrm{~N}$ & $\mathrm{~N}$ \\
\hline Giardina & 1992 & Italy & PNPF & $21 / 76$ & $15 / 6$ & 59.76 & $\mathrm{~N}$ \\
\hline Hutchinson & 1991 & England & PNPF & $35 / 35$ & $27 / 8$ & 46.5 & $\mathrm{~N}$ \\
\hline Marotta & 1991 & Japan & PNPF & $19 / 30$ & $\mathrm{~N}$ & $\mathrm{~N}$ & $\mathrm{~N}$ \\
\hline Takahashi & 1994 & Japan & PNPF & $67 / 47$ & $53 / 14$ & 63.7 & $\mathrm{~N}$ \\
\hline Tangkijvani & 1999 & Thailand & PNPF & $60 / 150$ & $50 / 10$ & 56.5 & $\mathrm{~N}$ \\
\hline
\end{tabular}

PNPF, PNPF endpoint method; CNPF, CNPF kinetic rate method; N, none; TNM, tumor node metastasis; BCLC, Barcelona clinic liver cancer; $M / F$, male/female.

Table 2: Subjects' raw data of the articles selected.

\begin{tabular}{lrrrr}
\hline Author $^{\text {a }}$ & \multicolumn{1}{c}{ TP } & FP & FN & TN \\
\hline El-houseini & 35 & 7 & 15 & 43 \\
El-houseini & 36 & 9 & 8 & 11 \\
Shao & 20 & 12 & 10 & 18 \\
Zhu, J & 64 & 18 & 49 & 84 \\
Zhang & 80 & 40 & 34 & 69 \\
Wang & 124 & 92 & 61 & 182 \\
Zhu, JN & 28 & 13 & 8 & 23 \\
Xing & 105 & 100 & 82 & 225 \\
Montaser & 36 & 1 & 4 & 39 \\
El-tayeh & 27 & 8 & 10 & 57 \\
Habachi & 61 & 45 & 25 & 44 \\
Mossad & 35 & 4 & 5 & 36 \\
Bukofzer & 54 & 19 & 18 & 45 \\
Giardina & 16 & 7 & 5 & 69 \\
Hutchinson & 21 & 12 & 14 & 23 \\
Marotta & 16 & 3 & 3 & 27 \\
Takahashi & 52 & 10 & 15 & 37 \\
Tangkijvani & 49 & 44 & 11 & 106 \\
\hline
\end{tabular}

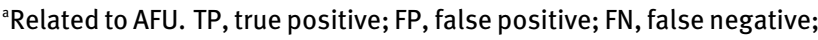
$\mathrm{TN}$, true negative.

\section{Quality of article}

The QUADAS-2 evaluation results are shown in Figure 2. It shows that the evaluation results on "risk of bias" are not ideal, while the evaluation results of "applicability concerns" are better. The main bias of these articles is concentrated on "patient selection" and "index test". In the "patient selection" part, only four articles used forwardlooking design. Twelve articles presented a experiment control design. In the part of "index test," 10 articles did not use any blinding method, and another article was not clear. On the other hand, the evaluation results of "reference standard" was deemed good, while the evaluation results of "flow and timing" in four articles were bad.

\section{Data analysis}

\section{Overall diagnostic accuracy of AFU and AFP for HCC}

The DOR forest plot of AFU in HCC diagnosis is shown in Figure 3. Figure 4 is ROC plot of AFU. The sensitivities of AFU and AFP were 0.7352 (0.6827, 0.7818) and 0.7501 $(0.6725,0.8144)$, respectively, while the specificities of AFU and AFP were $0.7681(0.6946,0.8283)$ and $0.8208(0.7586$, 0.8697) respectively. The DORs of AFU and AFP were 7.974 and 13.401, respectively. The sensitivity, specificity, and DOR of AFU and AFP are presented in Table 3.

\section{AFU heterogeneity test}

The purpose of studying heterogeneity is to identify the factors in the statistics that may affect the accuracy of estimations, and to assess whether the different studies combined are appropriate. Threshold effects are the most 


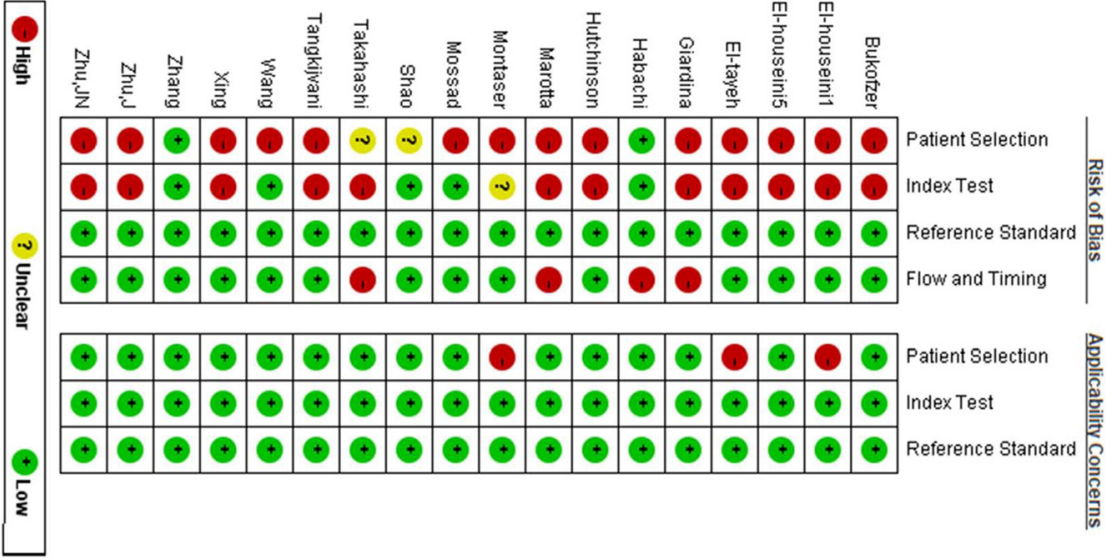

\section{} applicability concerns.

Figure 2: Summary of risk of bias and

Studies

El-house1 2001 El-house5 2005

Shao 2009

Zhu,J 2013

Zhang 2015

Wang 2014

Montaser 2012

El-tayeh 2012

Habach 2018

Mossad 2014

Bukofzer 1989

Giardina 1992

Hutchinson 1991

Marott 1991

Takahash 1994

Tangkijvani 1999

Xing 2019

Zhu,JN 2017

Overall $\left(I^{\wedge} 2=78.52 \%, P<0.001\right)$

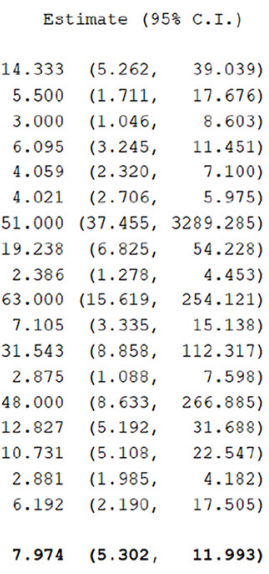

$(\mathrm{TP} * \mathrm{TN}) /(\mathrm{FP} * \mathrm{FN})$

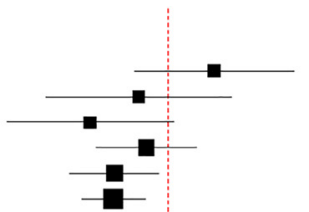

$22568 / 5612$

$1404 / 4$

$1539 / 80$

$2684 / 1125$

$1260 / 20$

$2430 / 342$

$1104 / 35$

$483 / 168$

$432 / 9$

$1924 / 150$

$5194 / 484$

$23625 / 8200$

$644 / 104$

$78448 / 18872$

\section{.}
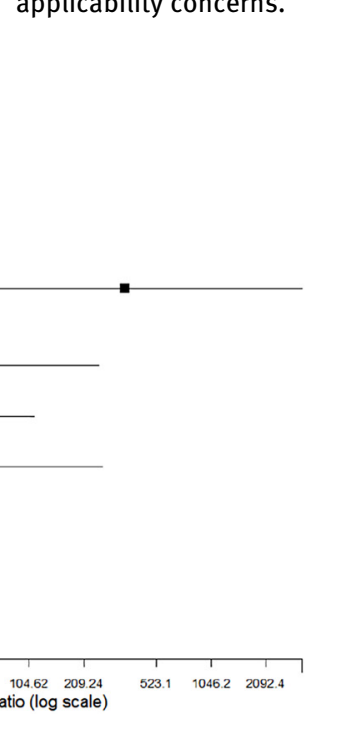

Figure 3: DOR forest plot for AFU.

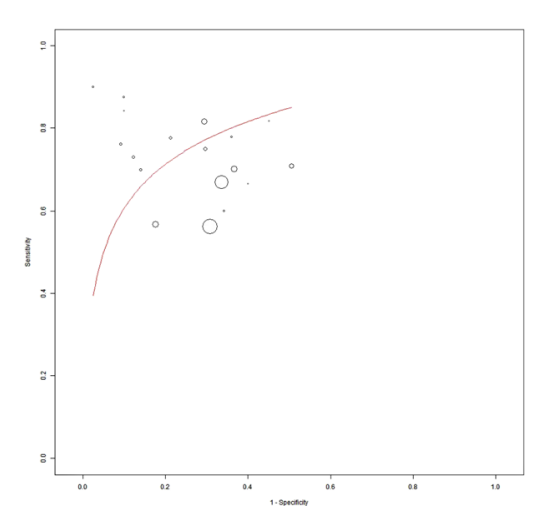

Figure 4: ROC diagram of AFU.

important cause of heterogeneity in diagnostic tests. In general, the threshold effect can be seen by the ROC plot. If the planar distribution displays a representative "shoulder arm shape," there is a threshold effect. In this study, the AFU's ROC plot did not display a "shoulder arm shape" (see Figure 4), indicating the absence of threshold effect. Also,
Table 3: DOR, sensitivity, and specificity of AFU and AFP.

\begin{tabular}{|c|c|c|}
\hline & AFU & AFP \\
\hline DOR $(95 \% \mathrm{Cl})$ & $\begin{array}{r}\text { 7.974(5.302, } \\
11.993)\end{array}$ & $\begin{array}{r}13.401(8.359 \\
21.483)\end{array}$ \\
\hline AUC & 0.7968 & 0.8451 \\
\hline Sensitivity $(95 \% \mathrm{Cl})$ & $\begin{array}{r}0.7352(0.6827 \\
0.7818)\end{array}$ & $\begin{array}{r}0.7501(0.6725 \\
0.8144)\end{array}$ \\
\hline Specificity $(95 \% \mathrm{Cl})$ & $\begin{array}{r}0.7681(0.6946 \\
0.8283)\end{array}$ & $\begin{array}{r}0.8208(0.7586 \\
0.8697)\end{array}$ \\
\hline $\mathrm{Tau}^{2}$ of DOR-Het. & 0.538 & 0.683 \\
\hline Q of DOR-Het. & 79.125 & 76.371 \\
\hline$I^{2}$ of DOR-Het. & $78.515 \%$ & $77.74 \%$ \\
\hline p of DOR-Het. & $<0.001$ & $<0.001$ \\
\hline Spearman(p) & $-0.396(0.103)$ & $0.406(0.095)$ \\
\hline$H=(Q / 17)^{0.5}$ & 2.1574 & 2.1195 \\
\hline
\end{tabular}

DOR, diagnostic odds ratio; AUC, area under the curve; Tau', estimate of between-study variance; Het, heterogeneity; $Q, Q$ statistic; $I^{2}$, inconsistency; $\mathrm{H}, \mathrm{H}$ statistic.

the Spearman correlation coefficient of $-0.396(\mathrm{p}=0.103)$ confirms that there is no threshold effect. 
However, in this analysis, the $\mathrm{I}^{2}$ of DOR-Het of AFU was $78.515 \%$, indicating that the heterogeneity is caused by non-threshold effects.

\section{Meta-regression for heterogeneity}

The heterogeneity was investigated by exploring the characteristics of these samples using meta-regression. Four DORs, which include HCC and control, gender, average age, and year, were examined. The results are that the $\mathrm{p}$-values of the four DORs are all $>0.1$, meaning that the four DORs have no heterogeneity.

We also performed a subgroup meta-analysis on the non-continuous data for the subgroup "country" and "test method". The results of this subgroup meta-analysis are shown in Table 4. The upper part of the table shows that three subgroups can be established, and only subgroup Egypt's $\mathrm{Q}=38.258, \mathrm{p}<0.001$, and $\mathrm{I}^{2}=86.76 \%$ indicate heterogeneity. However, $\mathrm{p}>0.05$ means no heterogeneity in the subgroup China and Japan and in these two subgroups, the detection effect of AFU is better.

The two most common detection methods, endpoint method and kinetic rate method, use different substrates to examine AFU activity. In the endpoint method, AFU activity is examined employing $p$-nitrophenyl- $\alpha$-L-furanoside (PNPF) as a colorimetric substrate [29-31]. The kinetic rate method typically uses 2-chloro-4-nitrophenyl- $\alpha$-glucopyranoside (CNPF) as a colorimetric substrate [32]. The lower part of Table 4 shows that two subgroups can be established. The subgroup PNPF's $Q=76.974, p<0.001$, and $\mathrm{I}^{2}=81.81 \%$ indicate heterogeneity, while $\mathrm{p}>0.05$ indicates no heterogeneity in the CNPF's subgroup.

\section{Discussion}

Hepatocellular carcinoma is the leading cause of death in patients with chronic liver disease. HCC is usually developed in an orderly progression from hepatitis to cirrhosis to early stage of cancer [33,34]. Serum marker detection shows many advantages over histopathological examination and there is a true need for sensitive and specific biomarkers. The serum AFP widely used for clinical detection of early HCC, and AFU has also been suggested as a serum marker for HCC as HCC patients have higher AFU concentrations and patients with benign liver disease have lower AFU levels [35].

This study explored the value of AFU in the diagnosis of HCC. To prevent confounding factors in the comparison we only chosen AFU and AFP in each patient. To improve accuracy, we analyzed only data from patients with chronic liver disease, precluding data from healthy people. We noticed the sensitivity, specificity, DOR, and AUC of AFU was lower than ones of AFP ( 0.7352 vs. $0.7501,0.7681$ vs. $0.8208,7.974$ vs. $13.401,0.7968$ vs. 0.8451$)$, respectively, but they are approximative, which indicates that the diagnostic value of AFU is comparable to one of AFP [17, 36]. Some papers reported that the combination of AFU and AFP may improve previous diagnostic value $[12,13,15,16$, 37]. In particular, El-Houseini (2001) reported that the simultaneous determination by AFP and AFU may improve the detection of HCC in cirrhotic patients negative for AFP. In addition, four articles show that AFU determination combined with other tumor markers increases the diagnostic accuracy of HCC [22, 23, 27, 37]. For example, Zhu et al. (2013) reported that the combined determination of GGT-II with AFU or AFP exhibited superior sensitivity and specificity for the diagnosis of HCC. However, many articles included in this analysis do not report a combined determination and the meta-analysis could not be conducted on the combined use of biomarkers for HCC diagnosis. Even though, a paper reported that the AUC of AFU combined with AFP was lower than that of AFP alone [17].

To explore heterogeneity, we excluded sequentially each article one by one and performed the meta-analyses. As none showed any significant change, heterogeneity is

Table 4: Results of subgroup meta-analysis based on DOR.

\begin{tabular}{|c|c|c|c|c|c|c|}
\hline & Subgroups & Studies & $\operatorname{DOR}(\mathrm{p})$ & $Q$ (df) & $p$ of Het & $I^{2}, \%$ \\
\hline \multirow[t]{7}{*}{ Country } & Subgroup Egypt & 6 & $17.002(<0.001)$ & $38.258(5)$ & $<0.001$ & 86.76 \\
\hline & Subgroup China & 6 & $3.839(<0.001)$ & $5.450(5)$ & 0.363 & 8.26 \\
\hline & Subgroup Japan & 2 & $20.120(<0.001)$ & 1.779 (1) & 0.182 & 43.78 \\
\hline & Subgroup South A & 1 & 7.105 (NA) & NA & NA & NA \\
\hline & Subgroup Italy & 1 & 31.543 (NA) & NA & NA & NA \\
\hline & Subgroup England & 1 & 2.875 (NA) & NA & NA & NA \\
\hline & Subgroup Thailand & 1 & 10.731 (NA) & NA & NA & NA \\
\hline \multirow[t]{2}{*}{ Method } & Subgroup PNPF & 15 & $9.316(<0.001)$ & $76.974(14)$ & $<0.001$ & 81.81 \\
\hline & Subgroup CNPF & 3 & $4.659(<0.001)$ & $1.516(2)$ & 0.469 & 0 \\
\hline
\end{tabular}

NA, not applicable; df, degrees of freedom; Het, heterogeneity. 
not caused by any individual research. Using a metaregression analysis, we found that country differences contribute to this heterogeneity. The subgroup analysis based on country demonstrates that only the Egypt subgroup shows heterogeneity except for the samples that could not be grouped, while the Chinese and the Japanese subgroups do not show heterogeneity, indicating better detection effects of AFU in these two subgroups.

Further, because many studies lack information on design, conduct, number of focal lesions in the liver, pathological stage, and focal size of the liver, we could not estimate if these variables cause bias. Wang et al. (2014) reported that preoperative AFU was an independent prognostic factor of overall survival. Patients with a preoperative AFU $>35 \mathrm{nmol} / \mathrm{mL} / \mathrm{min}$ had a lower recurrencefree survival rate and overall survival rate compared to patients with AFU $<35 \mathrm{nmol} / \mathrm{mL} / \mathrm{min}$, and they have a higher tendency to form macrovascular invasion. These data need to be confirmed by additional studies [24].

On the other hand, many studies lack detailed data on the pathological stage of HCC, limiting research on the relationship between AFU detection and HCC pathological stage.

\section{Conclusions}

AFU is close to AFP for the diagnosis of HCC, and the combined diagnostic value of AFP and AFU is worthy of further study, and the specific performance of AFU depending on a country remains to be clarified.

Acknowledgments: The authors thank Ms. Rihua Si, from Dept Lab Med, Affiliated Hosp 1, Nanjing Med Univ, for helpful comments and other works.

Research funding: None declared.

Author contributions: All authors have accepted responsibility for the entire content of this manuscript and approved its submission.

Competing interests: Authors state no conflict of interest. Ethical approval: The local Institutional Review Board deemed the study exempt from review.

\section{References}

1. Bray F, Ferlay J, Soerjomataram I, Siegel RL, Torre LA, Jemal A. Global cancer statistics 2018: GLOBOCAN estimates of incidence and mortality worldwide for 36 cancers in 185 countries. Ca Cancer J Clin 2018;68:394-424.

2. El-Serag HB. Epidemiology of viral hepatitis and hepatocellular carcinoma. Gastroenterology 2012;142:1264-73.
3. Ibrahim AM, Hashem ME, Mostafa EF, Refaey MM, Hamed EF, Ibrahim I, et al. Annexin A2 versus Afp as an efficient diagnostic serum marker for hepatocellular carcinoma. J Gastroenterol Hepatol Res 2013;212:780-5.

4. Masuzaki R, Karp SJ, Omata M. New serum markers of hepatocellular carcinoma. Semin Oncol 2012;39:434-9.

5. Ba MC, Long H, Tang YQ, Cui SZ. GP73 expression and its significance in the diagnosis of hepatocellular carcinoma: a review. Int J Clin Exp Pathol 2012;5:874-81.

6. Stefaniuk P, Cianciara J, Wiercinska-Drapalo A. Present and future possibilities for early diagnosis of hepatocellular carcinoma. World J Gastroenterol 2010;16:418-24.

7. Whiting PF, Rutjes AW, Westwood ME. QUADAS-2: a revised tool for the quality assessment of diagnostic accuracy studies. Ann Intern Med 2011;155:529-36.

8. The Nordic Cochrane Centre. Review manager (RevMan). Available from: https://community.cochrane.org/search/site/ RevMan [Accessed 1 Aug 2019].

9. Open Meta-analyst. Available from: http://www.cebm.brown. edu/openmeta/download.html [Accessed 1 Aug 2019].

10. Harbord RM, Whiting PM. Meta-analysis of diagnostic accuracy using hierarchical logistic regression. STATA J 2009;9: 211-29.

11. Bukofzer S, Stass PM, Kew MC. Alpha-L-fucosidase as a serum marker of hepatocellular carcinoma in southern African blacks. $\mathrm{Br}$ J Canc 1989;59:417-20.

12. El-Houseini ME, Mohammed MS, Elshemey WM. Enhanced detection of hepatocellular carcinoma. Can Contract 2005;12: 248-53.

13. El-Houseini ME. Serum alpha-L-fucosidase enzyme activity as a marker for hepatocellular carcinoma: comparison with AFU using ROC analysis. J Egypt Natl Canc Inst 2001;13:277-83.

14. El-Tayeh SF, Hussein TD, El-Houseini ME. Serological biomarkers of hepatocellular carcinoma in Egyptian patients. Dis Markers 2012;32:255-63.

15. Montaser MF, Sakr MA, Khalifa MO. Alpha-L-fucosidase as a tumour marker of hepatocellular carcinoma. Arab J Gastroenterol 2012;13:9-13.

16. Giardina MG, Matarazzo M, Varriale A. Serum alpha-L-fucosidase. A useful marker in the diagnosis of hepatocellular carcinoma. Cancer 1992;70:1044-8.

17. Xing H, Qiu H, Ding X, Han J, Li Z, Wu H. Clinical performance of $\alpha-\mathrm{l}$ fucosidase for early detection of hepatocellular carcinoma. Biomarkers Med 2019;13:545-55.

18. Zhu JN, Chen GD, Xu JY, Zhou X. Serum AFU, 5'-NT and AFP as biomarkers for primary hepatocellular carcinoma diagnosis. Open Med 2017;12:354-8.

19. Hutchinson WL, Johnson PJ, Du MQ. Serum and tissue alphaL-fucosidase activity in the pre-clinical and clinical stages of hepatocellular carcinoma. Clin Sci (Lond) 1991;81: 177-82.

20. Marotta F, Chui DH, Safran P, Zhang SC. Serum alphaL-fucosidase. A more sensitive marker for hepatocellular carcinoma? Dig Dis Sci 1991;36:993-7.

21. Shao Y. Union examination of AFP, AFU, AFPL3 and $\mathrm{y}$-GT in early diagnosis of primary liver cancer Acad J Xi'an Jiaot Univ 2009;2: 209-11.

22. Takahashi H, Saibara T, Iwamura S. Serum alpha-L-fucosidase activity and tumor size in hepatocellular carcinoma. Hepatology 1994;19:1414-7. 
23. Tangkijvanich P, Tosukhowong P, Bunyongyod P. AlphaL-fucosidase as a serum marker of hepatocellular carcinoma in Thailand. Southeast Asian J Trop Med Publ Health 1999;30:110-4.

24. Zhu J, Jiang F, Ni HB. Combined analysis of serum gammaglutamyl transferase isoenzyme II, alpha-L-fucosidase and alphafetoprotein detected using a commercial kit in the diagnosis of hepatocellular carcinoma. Exp Ther Med 2013;5: 89-94.

25. Zhang SY, Lin BD, Li BR. Evaluation of the diagnostic value of alpha-L-fucosidase, alpha-fetoprotein and thymidine kinase 1 with ROC and logistic regression for hepatocellular carcinoma. FEBS Open Bio 2015;5:240-4.

26. Wang K, Guo W, Li N, Shi J, Zhang C, Lau WY, et al. AlphaL-fucosidase as a prognostic indicator for hepatocellular carcinoma following hepatectomy: a large-scale, long-term study. Br J Canc. 2014;110:1811-9.

27. Habachi NA, El-Shayeb A, Mansour A, Zaghloul M. The validity of serum midkine, dickkopf- 1 and alpha-L-fucosidase as surrogate biomarkers for the diagnosis of hepatocellular carcinoma. J Hepatol 2018;68:S428.

28. Mossad NA, Mahmoud EH, Osman EA, Mahmoud SH, Shousha HI. Evaluation of squamous cell carcinoma antigen-immunoglobulin M complex (SCCA-IGM) and alpha-L-fucosidase (AFU) as novel diagnostic biomarkers for hepatocellular carcinoma. Tumor Biol 2014;35:11559-64.
29. Troost J, van der Heijden MC, Staal GE. Characterisation of alphaL-fucosidase from two different families with fucosidosis. Clin Chim Acta 1976;73:329-46.

30. Zielke K, Okada S, O'Brien JS. Fucosidosis: diagnosis by serum assay of alpha-L-fucosidase. J Lab Clin Med 1972;79:164-9.

31. Van Hoof F, Hers HG. The abnormalities of lysosomal enzymes in mucopolysaccharidoses. Eur J Biochem 1968;7:34-44.

32. Wang JJ, Cao EH. Rapid kinetic rate assay of the serum alpha-L fucosidase in patients with hepatocellular carcinoma by using a novel substrate. Clin Chim Acta 2004;347:103-9.

33. Coronel-Castillo CE, Qi XS, Contreras-Carmona J. Nonalcoholic fatty liver disease and nonalcoholic steatohepatitis in HIV infection: a metabolic approach of an infectious disease. Expet Rev Gastroenterol 2019;13:531-40.

34. Chandra K, Iqbal J. Protein carbonylation in freshly diagnosed hypothyroidism is independent of thyrotropin levels. J Lab Med 2018;42:177-81.

35. El-Shahawi MS, Othman AM, El-Houseini ME. Spectrofluorimetric method for measuring the activity of the enzyme alphaL-fucosidase using the ion associate of 2-chloro-4-nitrophenolrhodamine-B. Talanta 2009;80:19-23.

36. Ugur K, Aydin S, Donder E. Saliva and serum ghrelin and obestatin in iron deficiency anemia patients. J Lab Med 2018;42:183-8.

37. Malaguarnera G, Giordano M, Paladina I. Serum markers of hepatocellular carcinoma. Dig Dis Sci 2010;55:2744-55. 